\title{
Merger-driven evolution of the effective stellar initial mass function of massive early-type galaxies
}

\author{
Alessandro Sonnenfeld,,${ }^{1,2 \star}$ Carlo Nipoti,${ }^{3}$ and Tommaso Treu ${ }^{2}$ \\ ${ }^{1}$ Kavli IPMU (WPI), UTIAS, The University of Tokyo, Kashiwa, Chiba 277-8583, Japan \\ 2 Department of Physics and Astronomy, University of California, Los Angeles, 430 Portola Plaza, Los Angeles, CA 90025, USA \\ ${ }^{3}$ Department of Physics and Astronomy, Bologna University, viale Berti-Pichat 6/2, 40127 Bologna, Italy
}

8 October 2018

\begin{abstract}
The stellar initial mass function (IMF) of early-type galaxies is the combination of the IMF of the stellar population formed in-situ and that of accreted stellar populations. Using as an observable the effective IMF $\alpha_{\mathrm{IMF}}$, defined as the ratio between the true stellar mass of a galaxy and the stellar mass inferred assuming a Salpeter IMF, we present a theoretical model for its evolution as a result of dry mergers. We use a simple dry merger evolution model, based on cosmological $N$-body simulations, together with empirically motivated prescriptions for the IMF to make predictions for how the effective IMF of massive early-type galaxies changes from $z=2$ to $z=0$. We find that the IMF normalization of individual galaxies becomes lighter with time. At fixed velocity dispersion, $\alpha_{\mathrm{IMF}}$ is predicted to be constant with redshift. Current constraints on the evolution of the IMF are in slight tension with this prediction, even though systematic uncertainties prevent a conclusive statement. The correlation of $\alpha_{\mathrm{IMF}}$ with stellar mass becomes shallower with time, while the correlation between $\alpha_{\mathrm{IMF}}$ and velocity dispersion is mostly preserved by dry mergers. We also find that dry mergers can mix the dependence of the IMF on stellar mass and velocity dispersion, making it challenging to infer, from $z=0$ observations of global galactic properties, what is the quantity that is originally coupled with the IMF.
\end{abstract}

Key words: galaxies: elliptical and lenticular, $\mathrm{cD}$ - galaxies: evolution - galaxies: stellar content - stars: luminosity function, mass function

\section{INTRODUCTION}

Understanding the properties and the origin of the stellar initial mass function (IMF) is currently one of the biggest challenges in galaxy formation theory. Observational constraints on the IMF provide us with a puzzling scenario. On the one hand the IMF appears to be remarkably self-similar across different environments within the Milky Way (see e.g. Bastian et al. 2010; Offner 2015). On the other hand, the IMF in early-type galaxies is inferred to vary systematically as a function of mass or velocity dispersion (Treu et al. 2010; Cappellari et al. 2012; Conroy \& van Dokkum 2012; Dutton et al. 2012; Tortora et al. 2013; Spiniello et al. 2014; Posacki et al. 2015), although even for massive early-type galaxies we are still far from a clear picture (Smith et al. 2015). Efforts have been put into reproducing from a theoretical standpoint the observed IMF trends. However, despite recent progress (Hennebelle \& Chabrier 2011; Krumholz 2011; Hopkins 2012; Guszejnov et al. 2016), we

^ E-mail:alessandro.sonnenfeld@ipmu.jp still lack a coherent description of star formation across all environments.

One complication in comparing measurements of the IMF with models is that present-day stellar populations are ensembles of stars formed at different epochs in a range of environments. For massive early-type galaxies, a significant fraction of their present-day stellar mass is believed to be accreted from other systems (e.g. van Dokkum et al. 2010). If the IMF is not universal, then each accreted object will in general have a different IMF from the preexisting population of the central galaxy. The IMF of a massive galaxy at $z=0$ will then be the combination of the IMF of the stellar population formed in-situ and that of the accreted galaxies, possibly resulting in spatial gradients (Martín-Navarro et al. 2015; La Barbera et al. 2016). How does this "effective" IMF evolve in time? Answering this question and comparing the predictions to observations provides a new way to test galaxy formation and star formation models.

While the IMF is typically assumed as universal in cosmological simulations, there are studies of galaxy evo- 
lution based on semi-analytical models (SAMs) that allow for a non-universal IMF (Nagashima et al. 2005; Bekki 2013; Chattopadhyay et al. 2015; Gargiulo et al. 2015a; Fontanot et al. 2016). These works explore mostly the effect of the IMF on the chemical evolution of galaxies.

In order to isolate the effects of a varying IMF in the context of dry mergers, we adopt a simple model based on cosmological numerical simulations, galaxy-galaxy mergers simulations, and empirical prescriptions for the varying IMF. In practice, we use a simple prescription for assigning the starting $(z=2)$ IMF of an ensemble of galaxies and then evolve the stellar population of central galaxies by merging their stellar content with that of accreted satellites. We tune our model to match the correlation between IMF normalization and stellar mass observed at $z \sim 0$ and use it to make predictions on the stellar IMF of massive galaxies at higher redshifts. Though very simple in its construction, our model allows us to clearly isolate the effect of dry mergers, which are believed to represent the main growth mechanism of massive early-type galaxies at $z<2$, on the evolution of the IMF.

The paper is organized as follows. In Section 2 we describe our model for the IMF of $z=2$ galaxies and its evolution as a result of dry mergers. In Section 3 we present low- $z$ measurements of the IMF used to calibrate our model. In Section 4 we show our predictions on the time evolution of the IMF. We discuss our results in Section 5, while Section 6 concludes. We assume a flat $\Lambda \mathrm{CDM}$ cosmology with $H_{0}=70 \mathrm{~km} \mathrm{~s}^{-1} \mathrm{Mpc}^{-1}$ and $\Omega_{M}=0.3$. Throughout the paper velocity dispersions are expressed in units of $\mathrm{km} \mathrm{s}^{-1}$.

\section{THE MODEL}

\subsection{Parameters and notation}

Throughout our work we use the following quantities to describe the stellar content of our galaxies. We first define a true stellar mass, $M_{*}^{\text {true }}$. Then we introduce a Salpeter (Salpeter 1955) stellar mass, $M_{*}^{\text {Salp }}$, defined as the stellar mass one would infer by fitting a stellar population synthesis model based on a Salpeter IMF to broad-band photometric data. This quantity is typically used when observationally measuring stellar masses. We then consider the IMF mismatch parameter (Treu et al. 2010)

$$
\alpha_{\mathrm{IMF}}=\frac{M_{*}^{\text {true }}}{M_{*}^{\text {Salp }}} .
$$

Stellar populations with a more bottom-light IMF than a Salpeter IMF will have $\alpha_{\mathrm{IMF}}<1$. A Chabrier IMF for example corresponds typically to a value $\alpha_{\mathrm{IMF}} \approx 0.6$. As defined above, $\alpha_{\mathrm{IMF}}$ is a well-defined quantity also for galaxies that do not have a homogeneous stellar population, for example as a result of mergers. For typical cases $\alpha_{\text {IMF }}$ is mostly sensitive to the behaviour of the IMF at small masses, which dominate the mass budget but contribute little to the light. Depending on the parametrization it can be a probe of lowmass cutoff, and/or shape of the IMF. In extreme cases, $\alpha_{\text {IMF }}$ can be affected by the IMF at high masses, which, however, leaves an imprint not only on the mass-to-light ratio, but also on other phenomena, such as metal enrichment or supernovae rates. In this work, $\alpha_{\mathrm{IMF}}$ is the only parameter used to describe the IMF of a galaxy. Throughout our analysis we identify the terms effective $I M F$ and $I M F$ normalization with this quantity.

In addition to the stellar mass of a galaxy, we track its halo mass $M_{\mathrm{h}}$ and its central stellar velocity dispersion $\sigma$ : $M_{*}^{\text {true }}, M_{*}^{\text {Salp }}, M_{\mathrm{h}}$ and $\sigma$ are the only quantities that enter our model.

\subsection{The mock sample}

We generate a sample of 10000 halos at $z=2$ with masses drawn from the halo mass function described by Tinker et al. (2008), using an upper cut off at $\log M_{\mathrm{h}} / M_{\odot}<$ 13.5. We make this cut to focus on the regime of galaxy or galaxy group scale halos, where most observational constraints on the IMF are measured (Gavazzi et al. 2007), excluding cluster of galaxy environments (though IMF constraints are available for some brightest cluster galaxies; Newman et al. 2013). We then assign a Salpeter stellar mass to each halo using the stellar-to-halo mass relation (SHMR) from Leauthaud et al. (2012), including scatter. The Leauthaud et al. (2012) SHMR is originally expressed in terms of stellar masses calculated assuming a Chabrier IMF. For massive red galaxies, the assumption of a Salpeter IMF in stellar population synthesis measurements produces stellar masses larger than Chabrier by 0.25 dex, with negligible scatter (Auger et al. 2010b). Therefore we otain an SHMR in terms of $M_{*}^{\text {Salp }}$ by applying the transformation

$$
\log M_{*} \rightarrow \log M_{*}+0.25
$$

to the Leauthaud et al. (2012) SHMR.

We make an additional cut in stellar mass keeping only galaxies with $\log M_{*}^{\text {Salp }} / M_{\odot}>10.5$ and then select a random sample of 100 objects. Finally we assign central stellar velocity dispersions $\sigma$ assuming a power-law scaling with stellar mass,

$$
\log \sigma=\log \sigma_{0}+\beta_{\sigma}\left(\log M_{*}^{\text {Salp }}-11.5\right),
$$

with $\log \sigma_{0}=2.48$ and $\beta_{\sigma}=0.20$ and a scatter of 0.05 in $\log \sigma$. These values are such that the evolved population of mock galaxies reproduce the $M_{*}^{\text {Salp }}-\sigma$ relation measured at $z \sim 0$ by Auger et al. (2010b, see Section 2.4).

\subsection{The effective IMF}

The effectve IMF of massive galaxies has been shown to correlate with stellar velocity dispersion (e.g. Treu et al. 2010; Conroy \& van Dokkum 2012; Cappellari et al. 2012; La Barbera et al. 2013; Spiniello et al. 2014; Posacki et al. 2015), stellar mass (Auger et al. 2010a; Sonnenfeld et al. 2015), and stellar-mass density (Spiniello et al. 2015). In light of these observations, we adopt a relation of the following form for assigning the IMF to our model galaxies at $z=2$ :

$$
\log \alpha_{\mathrm{IMF}}=a_{*}\left(\log M_{*}^{\mathrm{Salp}}-\mu_{*}\right)+a_{\sigma}\left(\log \sigma-\mu_{\sigma}\right)+b,
$$

where $\mu_{*}$ and $\mu_{\sigma}$ are the average logarithms of stellar mass and velocity dispersion of the sample. We then choose two different sets of values for the parameters $a_{*}, a_{\sigma}$ and $b$. In the first prescription, labeled " $M_{*}$ model", we set $a_{*}=0.26$, $a_{\sigma}=0$ and $b=0.21$. For the second prescription, labeled " $\sigma$ model", we set $a_{*}=0, a_{\sigma}=1.20$ and $b=0.03$. In other 
terms, the first model is a power-law relation between IMF and stellar mass, with no residual dependence on $\sigma$, while vice-versa the IMF of the second model is set uniquely by the velocity dispersion. In each model, the values of the parameters have been tuned to approximately reproduce the low-z IMF measurements from S15. Details on these measurements are given in Section 3.

These prescriptions are purely empirically motivated. While there have been attempts at predicting the stellar IMF from first principles in terms of the global properties of a galaxy (e.g. Krumholz 2011; Hopkins 2012), implementing such models would require us to make additional assumptions on parameters of the star forming gas such as the pressure and the turbulence Mach number. Given the current uncertainty on the true mechanism determining the IMF, the benefits of employing such theoretically motivated recipes are modest and we therefore limit our model to simpler empirical recipes.

\subsection{The dry merger evolution}

Our central galaxies are evolved to $z \sim 0$ using the dry merger evolution model developed by Nipoti et al. (2012), with a few modifications. The method can be summarized as follows. For each central galaxy we compute the evolution in its halo mass using the following expression from Fakhouri et al. (2010), which is derived from the Millennium I and II simulations:

$$
\frac{\mathrm{d} \ln M_{\mathrm{h}}}{\mathrm{d} z}=-\frac{\dot{M}_{0}}{10^{12} M_{\odot} H_{0}} \frac{1+f z}{1+z}\left(\frac{M_{\mathrm{h}}}{10^{12} M_{\odot}}\right)^{g-1}
$$

with $\dot{M}_{0}=46.1 M_{\odot} \mathrm{yr}^{-1}, f=1.11$ and $g=1.1$. In this step we are assuming that the growth history of each halo in our sample is the same as the average growth history for halos of the same mass. We separate the growth in halo mass into smooth accretion and growth due to mergers. We estimate the latter with the following expression from Fakhouri et al. (2010),

$$
\frac{\mathrm{d}^{2} N_{\operatorname{merg}}}{\mathrm{d} z \mathrm{~d} \xi}\left(z, \xi, M_{\mathrm{h}}\right)=A\left(\frac{M_{\mathrm{h}}}{10^{12} M_{\odot}}\right)^{\alpha} \xi^{\beta} \exp \left[\left(\frac{\xi}{\tilde{\xi}}\right)^{\gamma}\right](1+z)^{\eta^{\prime}},
$$

which describes the number of mergers between halos of mass ratio $\xi$ per unit redshift interval. Following Fakhouri et al. (2010), we assume $A=0.0104, \tilde{\xi}=9.72 \times$ $10^{-3}, \alpha=0.133, \beta=-1.995, \gamma=0.263$ and $\eta^{\prime}=0.0993$. At each timestep $d z$, the change in halo mass due to mergers is then the integral over mergers of different halo mass ratios $\xi$

$$
\left[\frac{\mathrm{d} M_{\mathrm{h}}}{\mathrm{d} z}\right]_{\text {merg }}=-\int_{\xi_{\min }}^{1} M_{\mathrm{h}} \xi \frac{\mathrm{d}^{2} N_{\text {merg }}}{\mathrm{d} z \mathrm{~d} \xi} d \xi .
$$

We are assuming that only mergers with mass ratio larger than a minimum value $\xi_{\text {min }}$ contribute. This minimum value is set by the merging time-scale: mergers with very small values of $\xi$ have merging time-scales longer than the age of the Universe and therefore cannot contribute to the accreted mass. We fixed $\xi_{\min }=0.03$ following Nipoti et al. (2012). We tested for the impact of this choice on our study by varying $\xi_{\text {min }}$ between 0.01 and 0.1 , finding minimal differences in all the results.
We then calculate the growth in Salpeter stellar mass assuming that each accreted halo is associated with a Salpeter stellar mass given by the (redshift dependent) SHMR of Leauthaud et al. (2012), and that this stellar mass is also accreted by the central galaxy:

$$
\frac{\mathrm{d} M_{*}^{\text {Salp }}}{\mathrm{d} z}=-\int_{\xi_{\min }}^{1} \mathcal{R}_{*, h}\left(\xi M_{\mathrm{h}}, z\right) \xi M_{\mathrm{h}} \frac{\mathrm{d}^{2} N_{\text {merg }}}{\mathrm{d} z \mathrm{~d} \xi} d \xi,
$$

where $\mathcal{R}_{*, h}$ is the SHMR. With this equation we are assuming that each merger event brings in exactly the average amount of stellar mass for its halo mass: in other words we are neglecting scatter in the SHMR of the accreted satellites.

Each merger, in addition to increasing the mass of the galaxy, contributes to modify its velocity dispersion. Using the virial theorem under the assumption of parabolic orbits, Naab et al. (2009) showed that the ratio between the virial velocity dispersion after and before a merger event of mass ratio $\xi$ is given by

$$
\frac{\sigma_{v, f}^{2}}{\sigma_{v, i}^{2}}=\frac{1+\xi \epsilon}{1+\xi}
$$

where $\epsilon=\sigma_{v, a}^{2} / \sigma_{v, i}^{2}$ is the ratio between the velocity dispersion of the accreted object and that of the central galaxy, squared. When $\epsilon<1$ (as expected when $\xi<1$ ), $\sigma_{v, f}<\sigma_{v, i}$, so the velocity dispersion is expected to decrease as a consequence of a gas-poor, parabolic minor merger. We make the assumption that, for both the central and the satellite galaxies, the stellar velocity dispersion is proportional to their virial velocity dispersion $\sigma \propto \sigma_{v}$, so we can write the change in stellar central velocity dispersion due to mergers as

$$
\frac{\mathrm{d}^{2} \sigma^{2}}{\mathrm{~d} z \mathrm{~d} \xi}=\sigma^{2}\left[1-\frac{1+\xi \epsilon}{1+\xi}\right] \frac{\mathrm{d}^{2} N_{\mathrm{merg}}}{\mathrm{d} z \mathrm{~d} \xi} .
$$

We describe the velocity dispersion of the satellites with a relation between stellar mass and $\sigma$ of the form given by equation (3), with no scatter. The values of the coefficients $\sigma_{0}$ and $\beta_{\sigma}$ are determined by fitting equation (3) to the $M_{*}^{\text {Salp }}-\sigma$ relation of the central galaxies, and are updated at each timestep as the centrals evolve. In Fig. 1 we show the distribution in velocity dispersion of the mock galaxies as a function of stellar mass, at $z=0.2$ and $z=2$. We compare these values with the low- $z$ observations from Auger et al. (2010b) and the constraints at $z=2$ inferred by Mason et al. (2015). Our model tends to overpredict the velocity dispersions for a given stellar mass with respect to the values measured by Mason et al. (2015). The effects of this possible discrepancy on the results of this work are discussed in the next sections.

Finally, we need to describe the growth in true stellar mass for our centrals, which in turn requires to define the IMF of the accreted satellites. Similarly to the velocity dispersion case, we assign the IMF of satellites using equation (4), where the coefficients are determined at each timestep by fitting the distribution of IMF as a function of stellar mass and velocity dispersion of the centrals.

We do not keep track of the spatial distribution of stars within our galaxies. Although observations could in principle detect spatial variations in IMF within a galaxy (Martín-Navarro et al. 2015), provided that metallicity gradients are accurately accounted for (McConnell et al. 2016), 


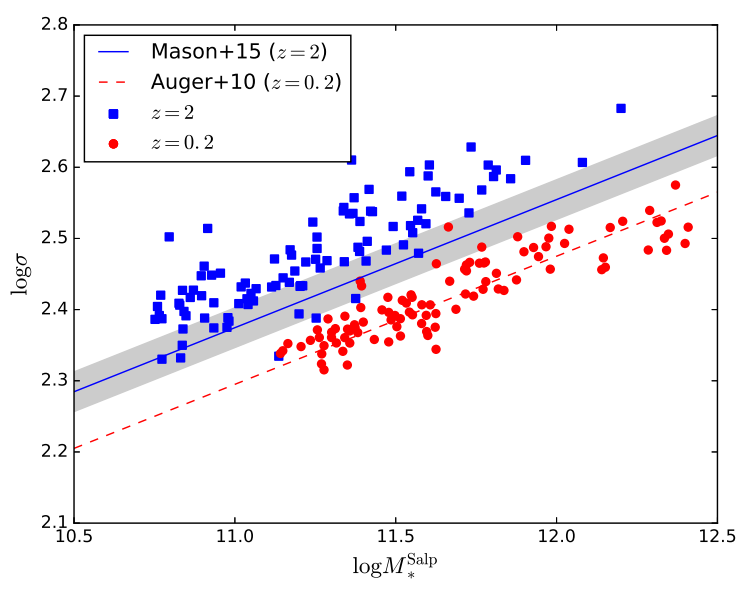

Figure 1. Velocity dispersion as a function of stellar mass of the " $M_{*}$ model" mock sample (the " $\sigma$ sample" behaves similarly), at $z=0.2$ (red circles) and $z=2$ (blue squares). Dashed line: best-fit $M_{*}^{\text {Salp }}-\sigma$ relation measured by Auger et al. (2010b) on a sample of strong lens early-type galaxies. Blue line: $M_{*}^{\text {Salp }}-\sigma$ relation at $z=2$ inferred by Mason et al. (2015). The $68 \%$ confidence region is marked by the shaded region.

and put interesting constraints on IMF differences between the in-situ population and that of the accreted stars, that is left for future work.

\section{REFERENCE IMF MEASUREMENTS}

The main goal of this work is to make predictions on the distribution of the IMF in massive galaxies as a function of redshift, using low-redshift observations as a reference point. To ensure self-consistency when comparing with wesults at high redshift, we choose the IMF measurements from Sonnenfeld et al. (2015, hereafter S15) to be such reference point. In this Section we summarize the main results of that work and carry out some additional analysis needed in order to calibrate our models to the observations.

S15 measured the effective stellar IMF for a sample of 80 early-type galaxies using strong lensing in combination with velocity dispersion data to constrain the stellar mass $M_{*}^{\text {true }}$ of each object. Measurements of $\alpha_{\text {IMF }}$ for individual objects are very noisy. To cope with the low signal-to-noise of individual measurements S15 combined them with a hierarchical Bayesian inference approach, in which they modeled the distribution of IMF normalization of the population of massive galaxies as a Gaussian and allowed the mean of such Gaussian to be a function of redshift, stellar mass and projected stellar mass density. The analysis yielded the detection of a positive trend between the effective IMF and stellar mass and no detection of a dependence on redshift or stellar mass density.

The S15 analysis did not explore dependences between velocity dispersion and IMF, which we consider in our model. Moreover, the relations between stellar mass and IMF explored by S15 were based on $M_{*}^{\text {true }}$, instead of $M_{*}^{\text {Salp }}$. For this reason we re-analyse here the $\mathrm{S} 15$ sample and fit for a dependence of the IMF on $M_{*}^{\text {Salp }}$ and $\sigma$, as well as redshift. We then describe the distribution in effective IMF of the S15 sample of galaxies as a Gaussian with mean

$a_{z}(z-0.3)+a_{*}\left(\log M_{*}^{\text {Salp }}-11.5\right)+a_{\sigma}(\log \sigma-2.4)+\log \alpha_{\mathrm{IMF}, 0}$

and dispersion $s$. The pivot points $z=0.3, \log M_{*}^{\text {Salp }}=11.5$ and $\log \sigma=2.4$ are very close to the median values of the corresponding quantities in the S15 sample. This allows us to minimize the covariance in the inference of the paramteres $a_{z}, a_{*}$ and $a_{\sigma}$. We fit for $a_{z}, a_{*}, a_{\sigma}, \alpha_{\mathrm{IMF}, 0}$ and $s$ using a similar hierarchical Bayesian inference method as the one used by S15. Details of the fitting procedure are given in the Appendix. The main assumptions of this model are: a fixed Navarro Frenk and White (Navarro et al. 1997, NFW) shape for the dark-matter distribution, a de Vaucouleurs (de Vaucouleurs 1948) profile with spatially constant mass to-light-ratio and isotropic velocity distribution for the stars.

In Fig. 2 we plot the posterior probability distribution for the parameters describing the IMF, marginalized over the other model parameters (which include parameters describing the dark-matter distribution). Consistently with the S15 analysis, we find an average IMF normalization slightly heavier than a Salpeter IMF $\left(\log \alpha_{\mathrm{IMF}, 0}=0.06 \pm 0.02\right)$. There is a strong degeneracy between the parameters $a_{*}$ and $a_{\sigma}$, meaning that the data can be described either with a dependence of the IMF on stellar mass or on velocity dispersion. This degeneracy is a consequence of the tight correlation between stellar mass and velocity dispersion in earlytype galaxies. Nevertheless the data, under the assumptions specified above and discussed in Appendix A, exclude a Universal IMF at the $3-\sigma$ level and show a trend of increasing IMF normalization with increasing mass and/or velocity dispersion.

Given that 1) our inference on the parameter $a_{*}$ is consistent with zero, 2) a description of the galaxy population in which the IMF depends simultaneously on velocity dispersion and stellar mass is not of trivial interpretation and 3) a large number of works in the literature focus on trends of the IMF with velocity dispersion alone, we repeat the analysis by fixing $a_{*}=0$. In this simpler description of the data, the IMF mismatch parameter is then distributed as a Gaussian with mean given by

$$
a_{z}(z-0.3)+a_{\sigma}(\log \sigma-2.4)+\log \alpha_{\mathrm{IMF}, 0}
$$

and dispersion $s$. The posterior probability distribution of the parameters of this model is plotted in Fig. 3.

\section{RESULTS}

\subsection{Evolution of the $\alpha_{\mathrm{IMF}}-M_{*}^{\text {Salp }}$ and $\alpha_{\mathrm{IMF}}-\sigma$ relations}

We evolved our mock population of galaxies from $z=2$ to $z=0.3$, the median redshift of the S15 sample and the redshift at which lensing constraints on the IMF are most robust. In Fig. 4 we plot the IMF mismatch parameter for our mock sample of galaxies at three different redshifts $(z=2$, $z=1$ and $z=0.3$ ), as a function of stellar mass and velocity dispersion, for each model. At each redshift snapshot and for each model we fit for a power-law dependence of the IMF on stellar mass

$$
\log \alpha_{\mathrm{IMF}}=c_{*}\left(\log M_{*}^{\text {Salp }}-11.5\right)+d_{*}
$$




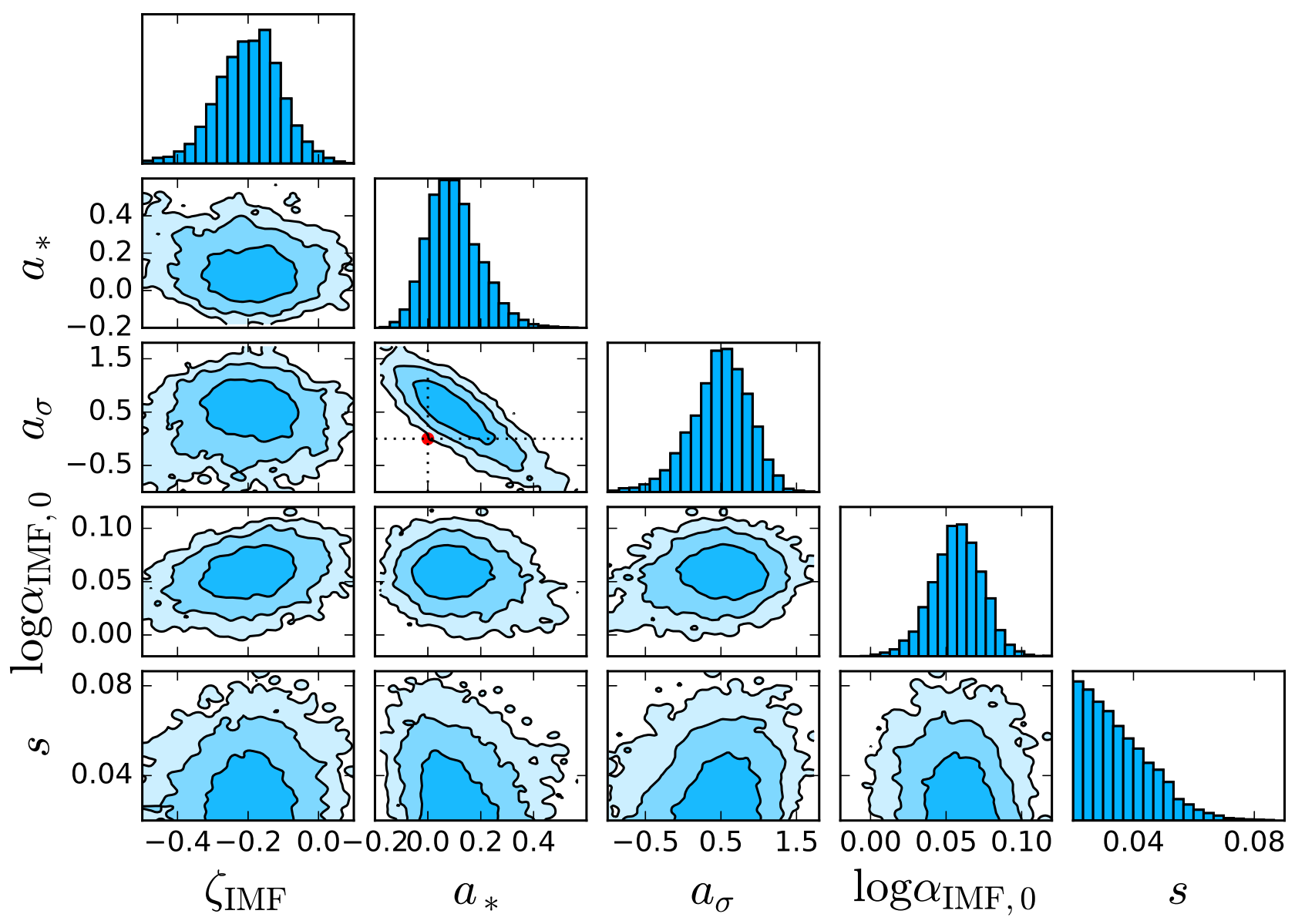

Figure 2. Posterior probability distribution of parameters describing the effective IMF of early-type galaxies, modeled as a Gaussian with mean given by equation (11) and dispersion $s$ and fitted to lensing and stellar kinematics observations of the S15 sample . Different levels represent the $68 \%, 95 \%$ and $99.7 \%$ enclosed probability regions. The red dot marks the values $a_{*}=0$ and $a_{\sigma}=0$, corresponding to a Universal IMF.

Table 1. Best-fit parameters of equation (13) and equation (14) to the mock data shown in Fig. 4. The third number in each set represents the residual scatter around the best fit relation.

\begin{tabular}{lcc}
\hline Data set & $\begin{array}{c}\text { Eq. 13 fit } \\
\left(c_{*}, d_{*}, s_{*}\right)\end{array}$ & $\begin{array}{c}\text { Eq. 14 fit } \\
\left(c_{\sigma}, d_{\sigma}, s_{\sigma}\right)\end{array}$ \\
\hline "M $M_{*}$ model" & $(0.26,0.21,0.00)$ & $(1.06,0.06,0.06)$ \\
$" M_{*}$ model" & $(0.21,0.13,0.02)$ & $(1.06,0.06,0.04)$ \\
"M $M_{*}$ model" & $(0.20,0.06,0.02)$ & $(1.08,0.05,0.04)$ \\
" $\sigma$ model" & $(0.24,0.19,0.05)$ & $(1.20,0.03,0.00)$ \\
" $\sigma$ model" & $(0.19,0.11,0.04)$ & $(1.06,0.05,0.01)$ \\
" $\sigma$ model" & $(0.18,0.05,0.03)$ & $(1.02,0.05,0.01)$ \\
\hline
\end{tabular}

and velocity dispersion

$$
\log \alpha_{\mathrm{IMF}}=c_{\sigma}(\log \sigma-2.4)+d_{\sigma} .
$$

Best-fit curves are overplotted in Fig. 4 and values of the parameters are reported in Table 1.

As can be seen by comparing the left hand panels in Fig. 4 with the right hand panels, correlations of the effective IMF with stellar mass correspond to similar correlations with velocity dispersion and vice-versa. This is again a consequence of the relatively tight correlation between galaxy stellar mass and velocity dispersion in the mock sample (and in observations; Fig. 1). By looking at the redshift evolution of our mock samples we can see that the IMF normalization of individual objects decreases with time. This can be easily understood: central galaxies grow by merging with smaller objects which, by construction, have a lighter IMF and therefore bring the effective IMF of the post-merger galaxy towards smaller values. Focusing on the left-hand side of Fig. 4 one can also notice how the most massive galaxies experience the largest decrease in IMF normalization. As a result, the correlation between stellar mass and effective IMF becomes shallower with time (the coefficient $c_{*}$ in equation (13) decreases with time). The reason for this tilt will be discussed in Section 5. A similar trend is seen for the correlation between $\sigma$ and $\alpha_{\text {IMF }}$ in the " $\sigma$ model" (bottom right panel of Fig. 4), becoming shallower with time. In contrast, for the " $M_{*}$ model" the original correlation between velocity dispersion and IMF is roughly preserved by dry mergers. 

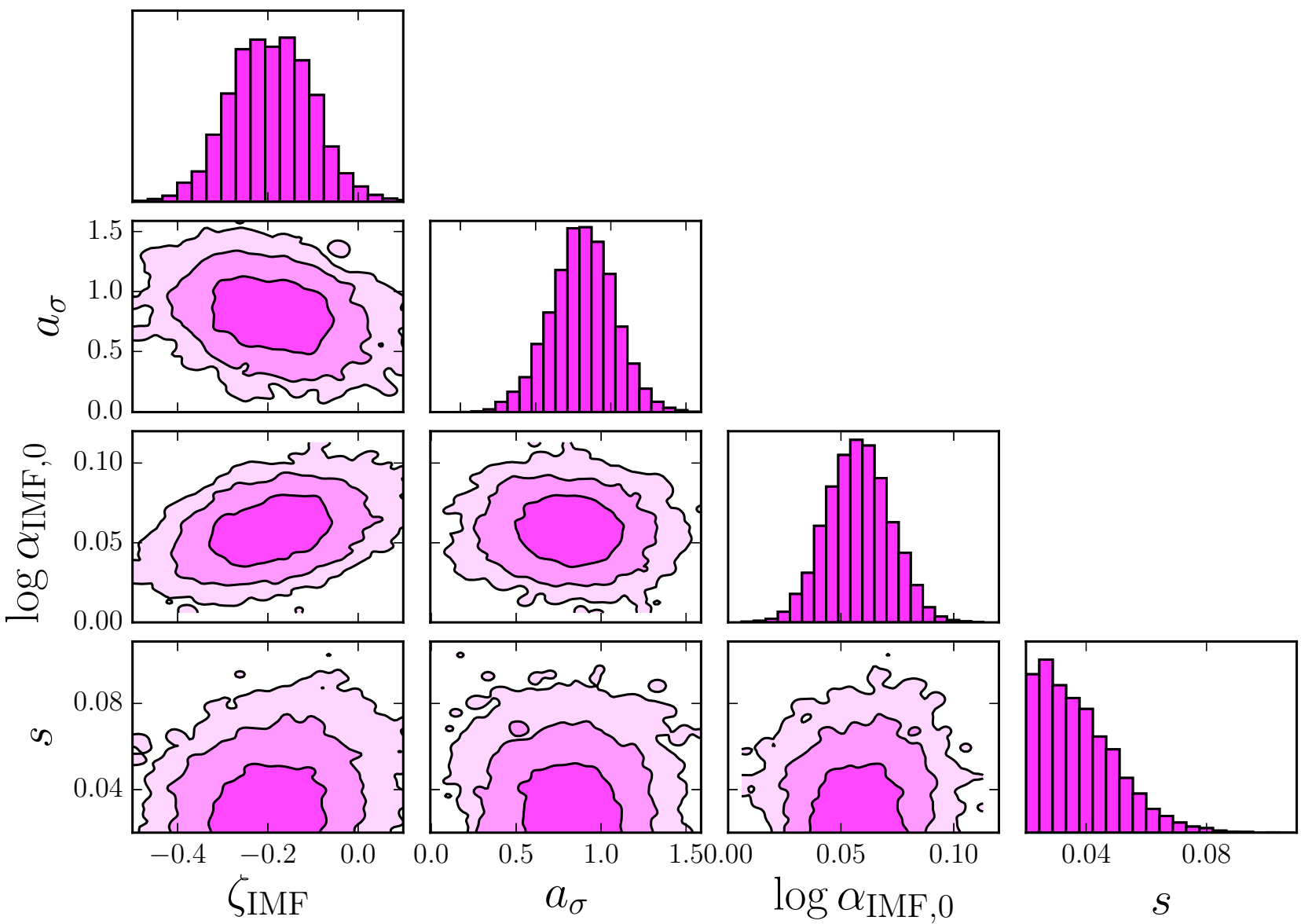

Figure 3. Posterior probability distribution of parameters describing the effective IMF of early-type galaxies, modeled as a Gaussian with mean given by equation (12) and dispersion $s$ and fitted to lensing and stellar kinematics observations of the S15 sample . Different levels represent the $68 \%, 95 \%$ and $99.7 \%$ enclosed probability regions.

While the mean trends between stellar mass or velocity dispersion and stellar IMF evolve very similarly for both models examined here, the two models differ in terms of scatter around these correlations. The " $M_{*}$ model" is initialized with zero scatter around the $M_{*}^{\text {Salp }}-\alpha_{\mathrm{IMF}}$ relation, but by $z=1$ a substantial scatter is introduced. For the " $\sigma$ model" however the relation between $\alpha_{\mathrm{IMF}}$ and $\sigma$ in place at $z=2$ remains remarkably tight down to $z=0.3$. This is a consequence of the different direction of the evolutionary tracks in $M_{*}^{\text {Salp }}-\alpha_{\text {IMF }}$ and $\sigma-\alpha_{\text {IMF }}$ space. Objects evolve roughly perpendicular to the $M_{*}^{\text {Salp }}-\alpha_{\text {IMF }}$ relation, quickly introducing scatter, while as discussed above the $\sigma-\alpha_{\mathrm{IMF}}$ relation is mostly preserved due to the coincidence between direction of evolution and correlation in $\sigma-\alpha_{\mathrm{IMF}}$ space. This prediction depends critically on our model for the evolution of the velocity dispersion with dry mergers, based on the assumption of parabolic orbits for the accreted satellites, which predicts steadily declining values of $\sigma$ with time for individual galaxies. Dark matter-only cosmological simulations show that the parabolic orbit approximation tends on average to underestimate the post-merger velocity dispersion (Posti et al. 2014). For instance, in the simple case in which $\sigma$ remains constant after each merger, objects would move vertically in the $\sigma-\alpha_{\text {IMF }}$ plane, qualitatively changing the right hand panels of Fig. 4.

\subsection{Evolution of the mock galaxies in the $\alpha_{\mathrm{IMF}}-M_{*}^{\text {Salp }}-\sigma$ space}

Fig. 4 only shows how the parameters $M_{*}^{\text {Salp }}$ and $\sigma$ correlate individually with the effective IMF. We now examine simultaneous dependences of the IMF on stellar mass and velocity dispersion, that is how $\alpha_{\text {IMF }}$ scales with $M_{*}^{\text {Salp }}$ at fixed $\sigma$ and viceversa. For each mock sample at each timestep we determine the average of the logarithm of the stellar mass and velocity dispersion, $\mu_{*}(z)$ and $\mu_{\sigma}(z)$, then fit equation (4) to the IMF distribution and plot the measured coefficients $a_{*}$ and $a_{\sigma}$ in Fig. 5.

The starting $(z=2)$ point in the $a_{*}-a_{\sigma}$ space is by construction very different for the two mock sets. For the " $M_{*}$ model" the initial dependence on stellar mass is in part converted into a dependence on velocity dispersion as a result of dry mergers. By contrast, the coefficients describing the $\sigma$ model at $z=0.3$ are very close to the $z=2$ values. The ini- 


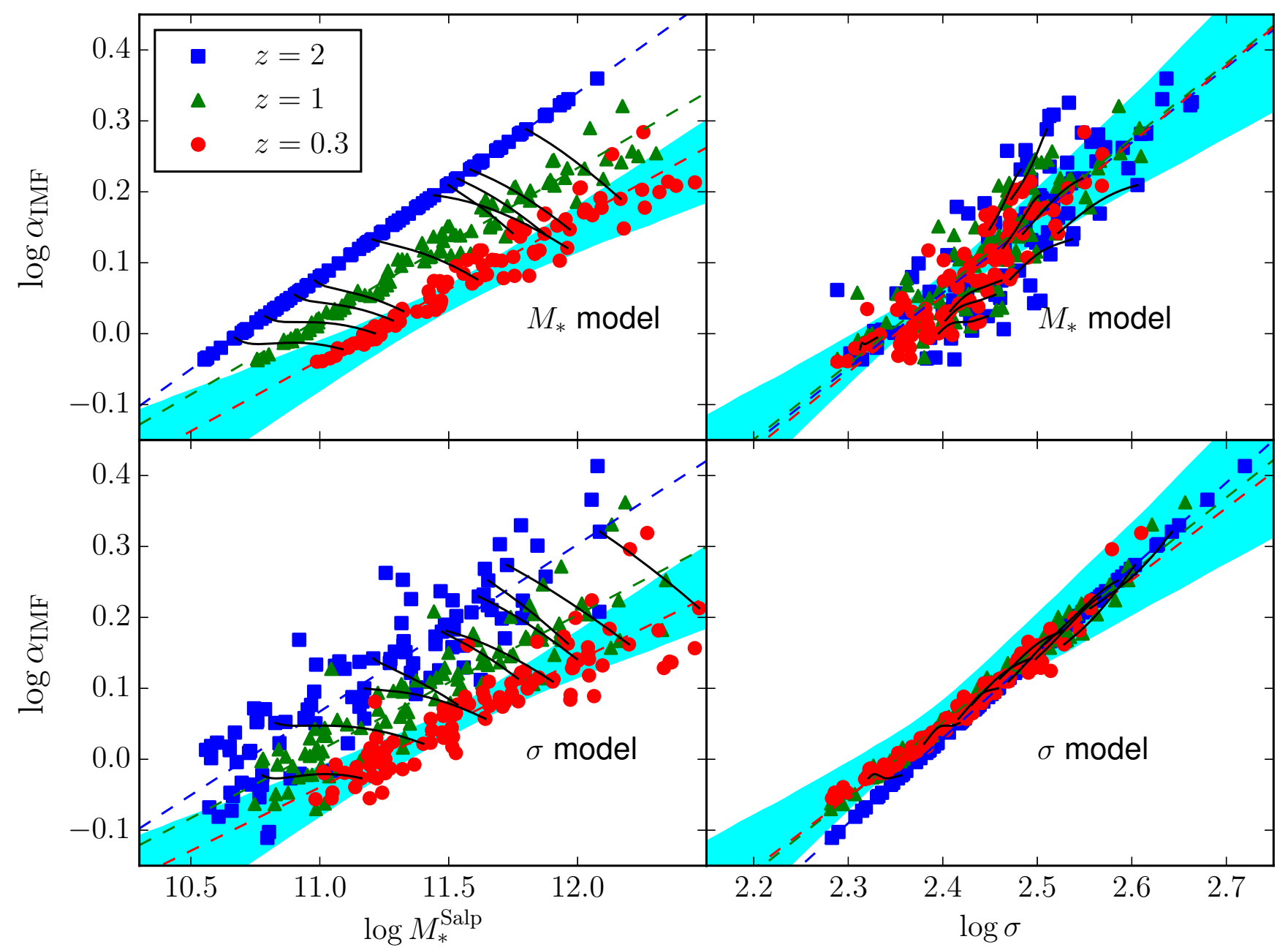

Figure 4. IMF mismatch parameter $\alpha_{\mathrm{IMF}}$ as a function of stellar mass (left panels) and central velocity dispersion (right panels) for the " $M_{*}$ model" (top panels) and " $\sigma$ model" (bottom panels) IMF recipes, at $z=2, z=1$ and $z=0.3$. Solid lines: Evolutionary tracks between $z=2$ and $z=0.3$ of a few representative galaxies. Dashed lines: fits of equation (13) and equation (14) to the data in each redshift snapshot. Shaded regions: Observational constraints from Section 3, obtained using data from S15 at $z=0.3$.

tial dependence of the IMF on velocity dispersion becomes slightly weaker while no significant dependence on stellar mass (at fixed velocity dispersion) is introduced. This is a result of dry mergers preserving the tight correlation between $\sigma$ and $\alpha_{\mathrm{IMF}}$, apparent in the bottom right panel of Fig. 4. As discussed above, this prediction relies in turn on the assumed evolution of the velocity dispersion. If we allow for a milder decline in velocity dispersion with dry mergers we observe a more significant mixing between stellar mass and velocity dispersion dependece of the IMF, the red dot corresponding to the " $\sigma$ model" in Fig. 5 approaching the corresponding dot of the " $M_{*}$ model".

\subsection{Average IMF normalization as a function of redshift at fixed velocity dispersion}

Finally we examine how the IMF normalization of the mock sample at fixed galaxy properties evolves in time and compare it with the S15 measurements. In Section 3 we presented two different descriptions of the IMF distribution: one in which $\alpha_{\mathrm{IMF}}$ scales with redshift, stellar mass and ve- locity dispersion (equation 11) and a simpler one in which the dependence on stellar mass is ignored (equation 12). Since, as shown in Fig. 5, the S15 data is unable to put interesting constraints on the separate dependence of the IMF on $M_{*}^{\text {Salp }}$ and $\sigma$, we adopt the latter description from now on and consider for simplicity only dependences of $\alpha_{\text {IMF }}$ on velocity dispersion and redshift.

In Fig. 6 we plot the mean IMF normalization for galaxies at $\log \sigma=2.4$ of the mock populations together with the value measured in the analysis of the S15 data. For the model curves, this is given by parameter $d_{\sigma}$ of equation (14), fitted for at each timestep. For the S15 measurements, the observational band is obtained by evaluating equation (12) at $\log \sigma=2.4$ at each redshift between $z=0$ and $z=0.8$ (there are no objects at $z>0.8$ in the S15 sample). We see a $2-\sigma$ discrepancy between both models and the S15 constraints.

We then compare our models with several sets of measurements from the literature. Conroy \& van Dokkum (2012) constrained the IMF of a sample of nearby massive ellipticals by fitting stellar population models to spectral 


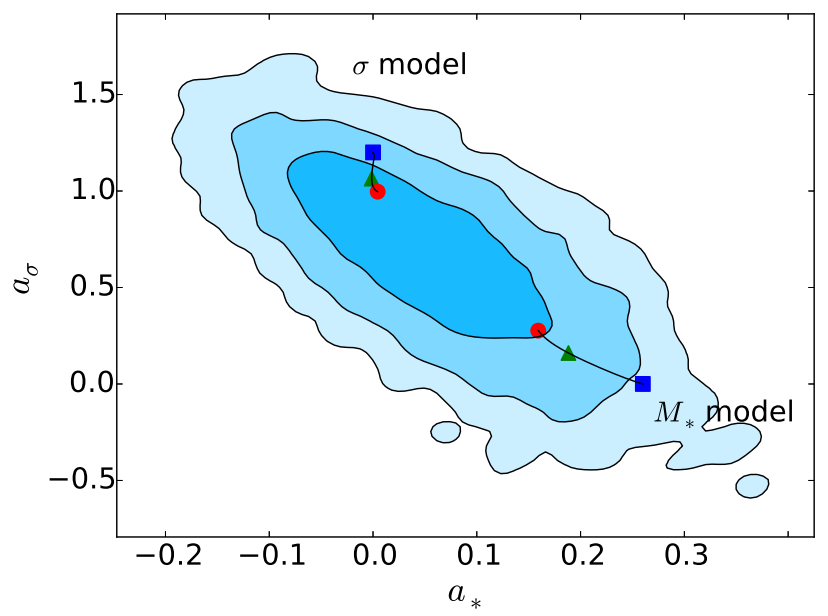

Figure 5. Coefficients $a_{*}$ and $a_{\sigma}$, describing the dependence of the effective IMF on stellar mass and velocity dispersion, obtained by fitting equation (4) to mock data generated with the " $M_{*}$ model" and " $\sigma$ model" prescriptions at each timestep. The shaded region marks the observational constraint at $z=0.3$ obtained in Section 3 from S15 data. Different contours mark regions of $68 \%$, $95 \%$ and $99.7 \%$ enclosed probability.

indices sensitive to the abundance of low-mass stars. They found a trend between IMF slope, and normalization, and stellar velocity dispersion. We took their measurements of the IMF normalization, converted them to our definition of $\alpha_{\mathrm{IMF}}$, then fitted for a power-law dependence between $\alpha_{\mathrm{IMF}}$ and $\sigma$ and plotted the value inferred at $\log \sigma=2.4$ in Fig. 6 . Using a similar technique, Spiniello et al. (2014) constrained the IMF of low redshift early-type galaxies by fitting stellar population models to a large sample of Sloan Digital Sky Survey spectra. They measured a positive trend between IMF normalization and galaxy velocity dispersion. In Fig. 6 we plot the value of $\alpha_{\mathrm{IMF}}$ inferred by Spiniello et al. (2014) for galaxies at $\log \sigma=2.4$.

These are all low-redshift measurements, plotted with the purpose of estimating the systematic uncertainty in the determination of $\alpha_{\text {IMF }}$ with different techniques. At higher redshift, Shetty \& Cappellari (2014) constrained the IMF of 68 galaxies at $z \sim 0.75$ from dynamical modeling of single aperture stellar kinematics data. Their analysis assumes that the total density profile follows the light distribution, neglecting the contribution of dark matter.

Although Shetty \& Cappellari (2014) report values of the IMF normalization and velocity dispersion for each object, we cannot use this information directly to fit for a trend between $\alpha_{\text {IMF }}$ and $\sigma$ because in dynamical analysis, unlike in the spectral fitting technique, the measurement uncertainty on the velocity dispersion is strongly correlated with that on the IMF. An accurate fit of $\alpha_{\text {IMF }}$ versus $\sigma$ would then require a full knowledge of the measurement uncertainty in the $\sigma-\alpha_{\text {IMF }}$ plane, which, unlike for the S15 sample, is not available. We then fix the slope of the $\sigma-\alpha_{\text {IMF }}$ relation to the value of $a_{\sigma}$ measured in the analysis of the S15 data and fit for the normalization of the IMF at $\log \sigma=2.4$ in the Shetty \& Cappellari (2014) sample. The resulting value of $\alpha_{\mathrm{IMF}}$ in slight tension with both the " $M_{*}$ model" and the " $\sigma$ model", though this tension is likely to disappear once the contribution from dark matter is taken into account.
At redshift higher than $z \sim 0.8$ there are no robust measurements of the IMF normalization. However we can put upper limits on the stellar mass, and the IMF, of galaxies with measured velocity dispersion using dynamical arguments. We can write the virial theorem for a stationary stellar system,

$$
K_{V} \sigma_{e 2}^{2}=G \frac{M}{R_{e}}
$$

where $M$ is the total (stellar plus dark-matter) mass, $R_{e}$ the projected half-light radius, $\sigma_{e 2}$ the luminosity-weighted line of sight velocity dispersion within a circular aperture of radius $R_{e} / 2$, and $K_{V}$ is a coefficient that takes into account the density profile of mass and light, geometrical effects and the distribution of stellar orbits. In the limit in which all the mass is in stars (no dark matter) $M=M_{*}^{\text {true }}$, so the virial coefficient $K_{V}$ can be used to put an upper limit on the stellar mass.

We then collect measurements of $\sigma_{e 2}$ in the redshift range $0.8<z<2$ from the literature. Following Mason et al. (2015) we consider measurements from van der Wel et al. (2008), Cappellari et al. (2009), Newman et al. (2010), Onodera et al. (2012), Bezanson et al. (2013), van de Sande et al. (2013), as well as measurements from Gargiulo et al. (2015b). We assume isotropic stellar velocity distribution, consistently with the analysis of the S15 data, and use the spherical Jeans equation to calculate $K_{V}$ for a mass-follows-light Sérsic (Sersic 1968) profile with structural parameters given by the observed values. The adopted values of $K_{V}$ range from 3.19 (corresponding to a Sérsic index $n=8.0$ ) to 7.18 (corresponding to $n=1.1)$. We solve for $M=M_{*}^{\text {true }}$ in equation (15) and divide the stellar mass by the value obtained from stellar population synthesis assuming a Salpeter IMF to obtain $\alpha_{\mathrm{IMF}}$. Finally, we correct these measurements to the pivot point $\log \sigma=2.4$ using the values of $a_{\sigma}$ measured in the analysis of S15 data. $1-\sigma$ upper limits (84\% enclosed probability) on $\log \alpha_{\mathrm{IMF}}$ for individual galaxies are plotted in Fig. 6. 39\% (34\%) of the objects have $1-\sigma$ upper limits below the " $M_{*}$ model" (" $\sigma$ model"), indicating tension between both models and observations. Part of this tension could simply be due to intrinsic scatter in the distribution of IMF in the population of galaxies. Although in Section 3 we constrained the intrinsic scatter to be below 0.08 in $\log \alpha_{\mathrm{IMF}}$, there are indications that the scatter in IMF might be larger (Smith \& Lucey 2013). Other possible causes for the discrepancy between models and observations are discussed in the next section.

\section{DISCUSSION}

\subsection{Comparison of models with observations}

Our model predicts that the effective IMF $\alpha_{\mathrm{IMF}}$ of massive early-type galaxies, at fixed velocity dispersion, should remain roughly constant with redshift. However, such a trend is not apparent from the few observational constraints on the IMF available at $z>0.3$ : in fact some data, including the S15 measurements used to calibrate our model at $z=0.3$, suggest that $\alpha_{\mathrm{IMF}}$ might be lower at higher $z$ (see also Tortora et al. 2014). A possible source for this tension 


\begin{tabular}{|c|c|c|c|c|c|}
\hline 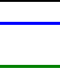 & $\begin{array}{l}M_{*} \text { model } \\
\sigma \text { model }\end{array}$ & $\nabla$ & $\begin{array}{l}\text { van de Sande et al. } 2013 \\
\text { Bezanson et al. } 2013\end{array}$ & $\underset{\nabla}{\nabla}$ & $\begin{array}{l}\text { van der Wel et al. } 2008 \\
\text { Spiniello et al. } 2014\end{array}$ \\
\hline & S15 & $\boldsymbol{\nabla}$ & Onodera et al. 2012 & ठ & Conroy \& van Dokkum 2012 \\
\hline$\nabla$ & Gargiulo et al. (2014) & $\nabla$ & Cappellari et al. 2009 & $\Phi$ & Shetty \& Cappellari 2014 \\
\hline$\nabla$ & Belli et al. (2014) & $\nabla$ & Newman et al. 2010 & & \\
\hline
\end{tabular}

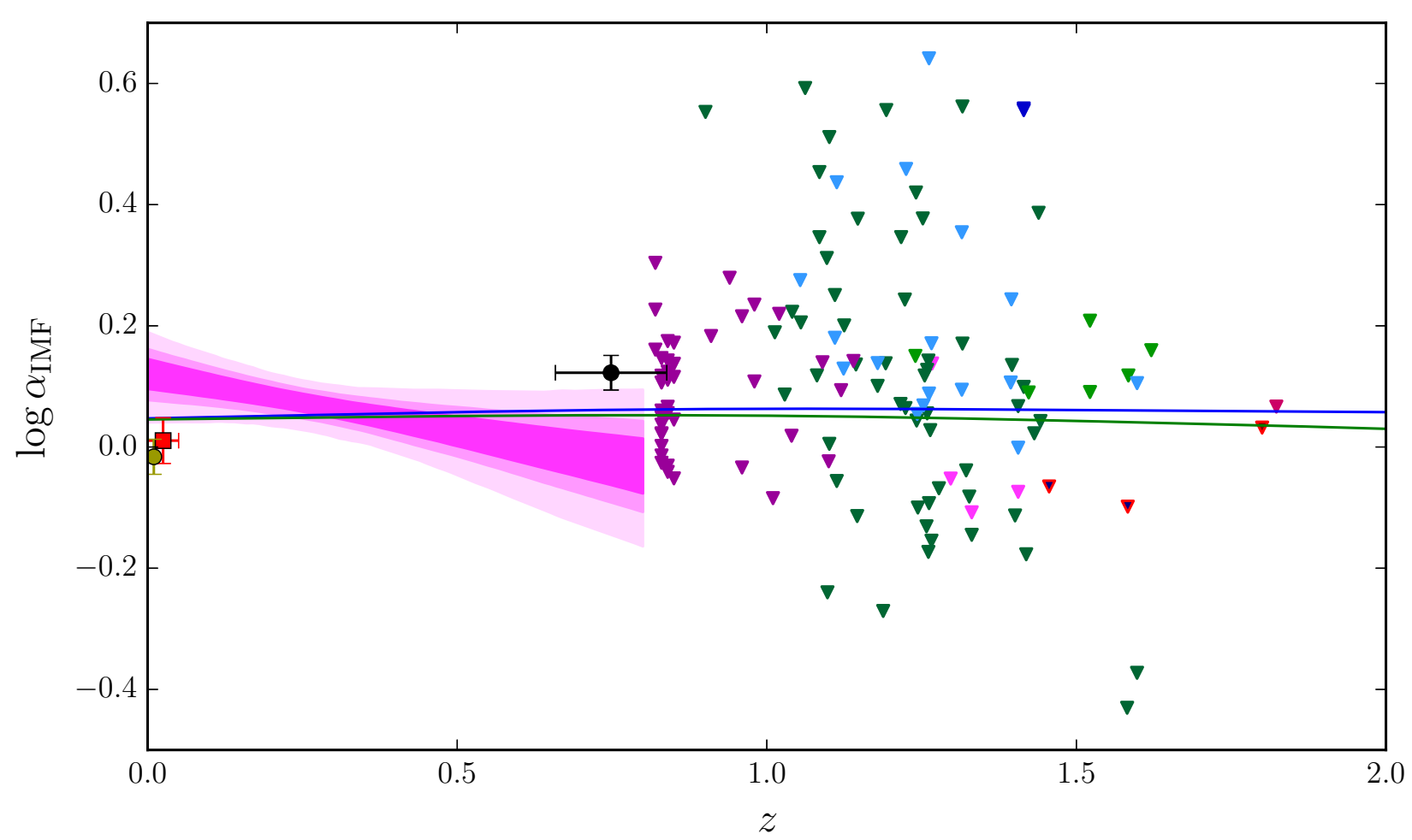

Figure 6. Solid lines: Average effective IMF normalization for galaxies at $\log \sigma=2.4$ of the mock samples, parameter $d_{\sigma}$ in equation (14), as a function of redshift. Shaded regions: Observational constraints from the analysis of S15 data carried out in Section 3, obtained by evaluating equation (12) at $\log \sigma=2.4$. Different levels mark the $68 \%, 95 \%$ and $99.7 \%$ enclosed probability regions. Points with error bars: Average IMF normalization at $\log \sigma=2.4$ inferred by Conroy \& van Dokkum (2012), Spiniello et al. (2014) and Shetty \& Cappellari (2014). Horizontal error bars indicate the range in redshift of the galaxies within the samples. Triangles: upper limit estimates of $\alpha_{\text {IMF }}$ from isotropic mass-follows-light spherical Jeans dynamical models applied to velocity dispersion measurements from the literature, corrected to the pivot point assuming the value of $a_{\sigma}$ measured in the S15 analysis. The triangle marks the $1-\sigma$ (84 percentile) limit.

is the treatment of the dark-matter component in the analysis of the S15 data, in which we fixed the shape of the dark-matter density distribution to an NFW profile (see the Appendix). The dark-matter slope is however highly degenerate with the IMF (see e.g. Auger et al. 2010b). In particular, if the dark-matter profile is getting steeper with time the IMF normalization inferred at low redshift would decrease, bringing the model in better agreement with the measurements. However such a hypothesis is difficult to verify with the current data.

Although the discrepancy between the models and the S15 measurements is within $3-\sigma$, a similar tension is seen in the comparison with dynamical estimates on the IMF at $z>1$. Dark matter cannot be a source of bias in this case because its contribution is ignored in our treatment of high redshift measurements.

Another possible source of error that could be affecting the measurements differently at different redshifts is the presence of spatial gradients in the IMF. As shown by Newman et al. (2015), an IMF that becomes less heavy at increasing distance from the center of a galaxy would bias lensing and dynamical studies that assume a spatially constant IMF (such as the observations presented here) towards a globally heavier IMF. A radial gradient in IMF could be created by dry mergers if the newly accreted material has a more extended distribution than the preexisting stellar distribution. Indeed, minor dry mergers are predicted to build up an extended envelope of stars (Naab et al. 2009; Hopkins et al. 2010) which, in the context of our model, would have a lighter IMF compared to the central parts. Such an extended envelope of stars with lighter IMF would grow in time, producing a stronger bias towards a heavier inferred IMF in low redshift galaxies. Martín-Navarro et al. (2015) and La Barbera et al. (2016) claimed a detection of a spatial variation of the IMF, heavier in the center, in three massive early-type galaxies. Testing whether such a scenario 
could be the cause of the apparent evolution in IMF would require a much more complex model than the one explored in this work, and we therefore leave it to future study.

Assuming that the tension between model and data is real and not the result of systematic errors or selection effects, our simple scenario based on dry mergers must be modified. In a previous work we showed how a very similar dry merger evolution model to the one presented here is unable to reproduce both the size evolution and the evolution in the slope of the density profile of massive galaxies at $z<1$ (Sonnenfeld et al. 2014). The model predicted density slopes that become shallower with time, at odds with lensing constraints. As a solution we proposed a scenario in which a small fraction (about 10\%) of the accreted baryonic mass is in the form of gas that falls to the center of the galaxy causing the density profile to steepen and the velocity dispersion to increase as a result of adiabatic contraction.

Such a scenario would modify the predicted IMF evolution in two ways: with the addition of a population of stars formed in-situ and by modifying the evolution of the velocity dispersion. Contributing with less than $10 \%$ of the final mass, the newly formed stars would not change significantly the effective IMF of a galaxy unless their IMF normalization differs by a factor of a few with respect to that of the accreted satellites, which seems unplausible. However, a central core of stars with a different IMF from the preexisting population would create a radial gradient in the IMF which, as discussed above, could be a source of bias for dynamical measurements. The increase in velocity dispersion caused by the adiabatic contraction from the infall of gas would improve the agreement with the observed evolution of the $M_{*}^{\text {Salp }}-\sigma$ relation. We have shown in Fig. 1 how our model tends to overpredict the average velocity dispersion at $z=2$ with respect to observations, meaning that a slower decline in velocity dispersion is required to precisely match both $z=0$ and $z=2$ velocity dispersion measurements. However, in a scenario in which the velocity dispersion evolves more slowly the $\sigma-\alpha_{\mathrm{IMF}}$ correlation will not be preserved, as predicted in our purely dry-merger model (see right-hand panel of Fig. 4), but will become shallower with time. This means that at fixed $\sigma$ the IMF will be heavier at larger $z$, increasing the tension between model and $z>1$ data.

Finally, a possible solution to the discrepancy would be to allow for completely different IMF distributions between centrals and satellites. Our model assumes that the scaling relation between the effective IMF of accreted galaxies and their galaxy properties is the same as that of centrals, extrapolated to low masses. If we allow, for instance, the IMF normalization of satellites to be on average heavier than that of centrals, individual galaxies would evolve towards a heavier effective IMF with time, in the direction suggested by the data. There are currently no robust observations through which we can test this hypothesis.

\subsection{IMF evolution and average merger mass ratio}

A second prediction of our model is that the deacrease of the IMF mismatch parameter with time is stronger for the more massive objects. This is shown by the evolution in the tilt of the $\alpha_{\mathrm{IMF}}-M_{*}^{\text {Salp }}$ correlation with redshift seen in the left hand column of Fig. 4. To understand the origin of this effect, in Fig. 7 we plot the fractional change in effective
IMF between $z=2$ and $z=0, \Delta \log \alpha_{\mathrm{IMF}}=\log \alpha_{\mathrm{IMF}}(z=$ $0)-\log \alpha_{\mathrm{IMF}}(z=2)$ of our mock galaxies together with key quantities such as the initial halo mass and initial stellar mass and accreted stellar mass. The effective IMF of individual galaxies in our sample generally decreases with time $\left(\Delta \log \alpha_{\mathrm{IMF}}<0\right)$. This is easily understood, as by definition central galaxies merge with less massive systems that, in our model, have a lighter IMF. In order to produce a dependence of the IMF on mass that becomes shallower with time it is necessary for the more massive systems to have a stronger decrease in their IMF with time (more negative values of $\left.\Delta \log \alpha_{\mathrm{IMF}}\right)$. As can be seen in the bottom left panel of Fig. 7, this is indeed the case.

Differences in $\Delta \log \alpha_{\mathrm{IMF}}$ reflect differences in merger history. Since mergers tend to decrease the effective IMF normalization, we expect galaxies with the highest increase in stellar mass to also show the largest changes in effective IMF. However this is not observed to be the case, as can be seen from Fig. 7 (fourth row, third column). If the amount of accretion does not play a central role in setting the final IMF, then the different change in IMF between low-mass and high-mass systems must be set by differences in the type of mergers. To quantify this, we measure the accreted stellar mass-weighted merger mass ratio, $\langle\xi\rangle_{*}$, defined as the average over all mergers between $z=2$ and $z=0$ of the mass ratio between the accreted satellites and the central halo weighted by the accreted stellar mass with each merger:

$$
\langle\xi\rangle_{*}=\frac{\int d z \int d \xi \xi f(\xi, z)}{\int d z \int d \xi f(\xi, z)},
$$

with

$$
f(\xi, z) \equiv \frac{\mathrm{d}^{2} N_{\text {merg }}}{\mathrm{d} z \mathrm{~d} \xi} \mathcal{R}_{*, h}\left(\xi M_{\mathrm{h}}, z\right) \xi M_{\mathrm{h}}
$$

$\langle\xi\rangle_{*}$ is plotted in Fig. 7. We see that $\langle\xi\rangle_{*}$ increases for decreasing halo and stellar mass. This means that for the most massive galaxies the bulk of the accreted stellar mass is brought in through mergers of relatively small mass ratio, while for less massive galaxies major mergers play a more important role. Given our IMF prescription, where the heaviness of the IMF of satellites increases with mass, minor mergers produce a stronger decrease in the effective IMF of a galaxy than major mergers at fixed accreted stellar mass. This is seen in the bottom right panel of Fig. 7, where $\langle\xi\rangle_{*}$ correlates with the change in effective IMF. We then conclude that the shallowing of the $M_{*}^{\text {Salp }}-\alpha_{\text {IMF }}$ or $\sigma-\alpha_{\text {IMF }}$ trends with time seen in our Fig. 4 is a result of the different relative importance of major and minor mergers in systems of different mass.

\subsection{Dependence of the IMF on global galaxy properties}

Another prediction of our model is the mixing between stellar mass and velocity dispersion dependence of the IMF shown in Fig. 5. The dependence of velocity dispersion, $a_{\sigma}$, seen at $z=0$ in the " $M_{*}$ model" can be easily understood. The " $M_{*}$ model" is initialized with zero scatter around the $M_{*}^{\text {Salp }}-\alpha_{\text {IMF }}$ relation at $z=2$. Since then, individual galaxies evolve roughly perpendicularly to the $M_{*}^{\text {Salp }}-\alpha_{\mathrm{IMF}}$ relation (left hand panels of Fig. 4), so that by $z=1$ a significant scatter is introduced. At that point a pure dependence of the 


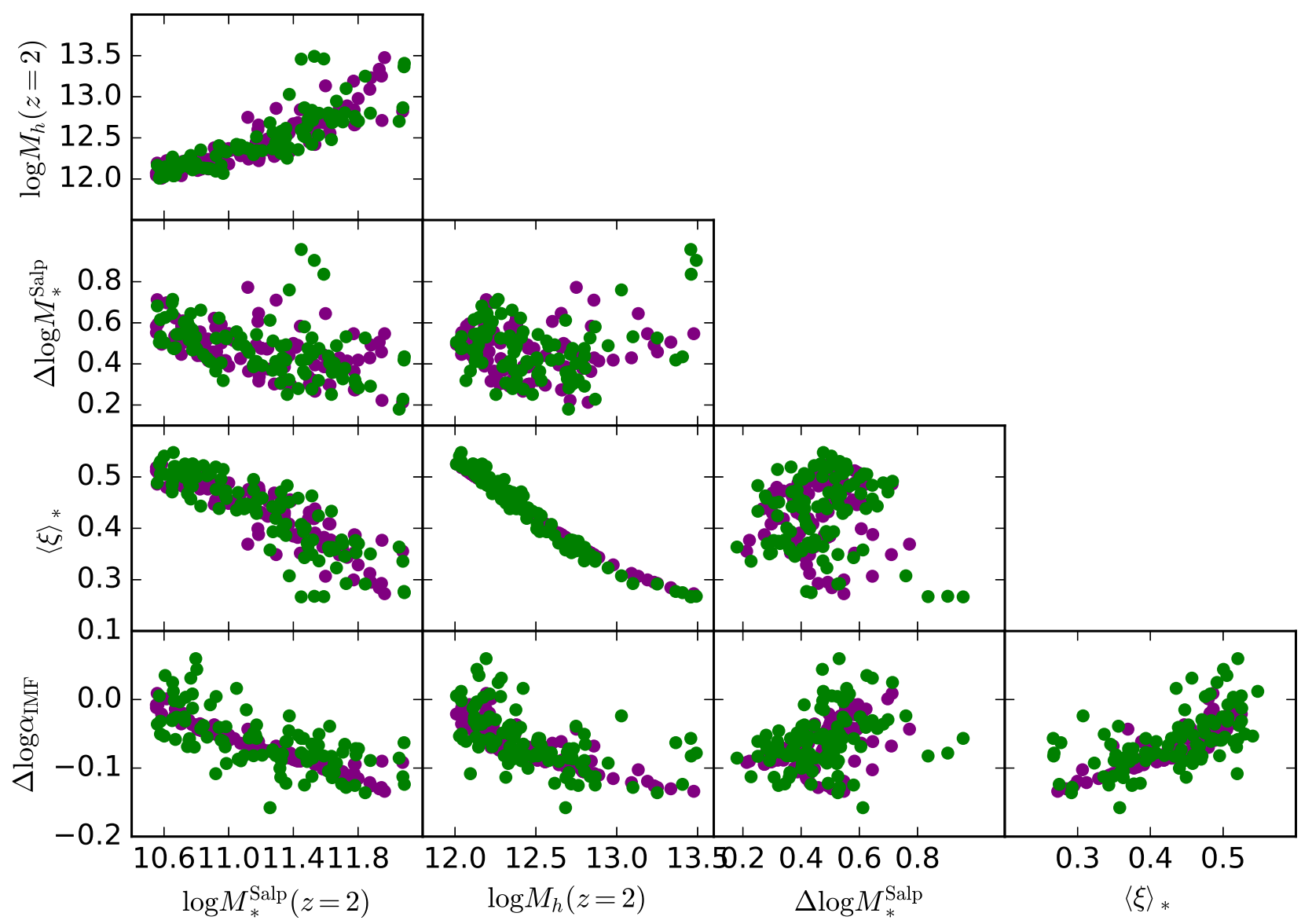

Figure 7. Distribution in stellar mass $M_{*}^{\text {Salp }}$ and halo mass $M_{\mathrm{h}}$ at $z=2$, fractional variation in stellar mass $\Delta \log M_{*}^{\text {Salp }}$ and effective IMF $\Delta \log \alpha_{\mathrm{IMF}}$ between $z=0$ and $z=2$, and stellar mass-weighted merger mass ratio $\langle\xi\rangle_{*}$, as defined in equation (16). Purple dots: " $M_{*}$ model". Green dots: " $\sigma$ model".

IMF on stellar mass is no longer a good description of the model, and a mixed dependence on both $M_{*}$ and $\sigma$ is preferred. In contrast in the " $\sigma$ model" galaxies evolve parallel to the $\sigma-\alpha_{\text {IMF }}$ relation in place at $z=2$ and very little scatter is added down to $z=0.3$, so that measuring the scatter in IMF as a function of $z$ is potentially a powerful diagnostic. No additional dependence on $M_{*}$ is required to describe the data, hence the very small change in the parameters $a_{*}$ and $a_{\sigma}$ for this model. As discussed in Section 4 however, this prediction relies on the assumption of parabolic orbits for the accreted satellites. If we allow for a milder decrease in velocity dispersion with dry mergers, we find a larger increase in the parameter $a_{*}$ and a correspondingly larger decrease in $a_{\sigma}$ for the " $\sigma$ model". Because of the relatively rapid change in the coefficients $a_{*}$ and $a_{\sigma}$ with time it is very difficult to determine from $z \sim 0$ observations which quantity between $\sigma$ and $M_{*}$ is the IMF fundamentally dependent on. Current constraints from lensing and stellar dynamics are unable to differentiate between the two models (see Fig. 5).

An important assumption in our model is that all of the growth in stellar mass between $z=2$ and $z=0$ is due to dry mergers. In other terms, our model only applies to the population of quiescent galaxies at $z=2$. This assumption complicates the comparison between predictions from our model and observations, because the number density of quiescent galaxies is observed to increase substantially with time, in particular at $z>1$ (e.g. Ilbert et al. 2013; Cassata et al. 2013). Depending on what the IMF of the newly quenched object is, some results might change as a result of the so-called progenitor bias. However: predictions on the $z<1$ evolution for the high-mass end of the galaxy distribution should still hold, since the number density of quiescent galaxies shows little evolution in that region of the parameter space (López-Sanjuan et al. 2012).

\section{CONCLUSIONS}

Using empirically motivated recipes for describing the IMF of massive galaxies together with the dry merger evolution model developed by Nipoti et al. (2012) we studied the time evolution of the effective IMF, defined as the ratio between the true stellar mass and the stellar mass one would infer assuming a Salpeter IMF, of a population of massive galaxies 
from $z=2$ to $z=0$. The models are set up to match the observed correlations between IMF and stellar mass and velocity dispersion in massive early-type galaxies at $z \sim 0.3$.

Our model predicts a decrease in the effective IMF of individual objects with time as galaxies merge with systems with a lighter IMF. This is seen in the evolutionary tracks in Fig. 4. Trends between stellar mass and velocity dispersion are qualitatively preserved by dry mergers, but the slope of the correlation between stellar mass and effective IMF becomes less steep with time.

At fixed velocity dispersion, the population of galaxies evolves with a constant or increasing IMF normalization at increasing $z$. This prediction appears to be in slight tension with the few observational constraints on the IMF available at $z>0.3$. However, there are systematic effects that could be affecting observations the imporance of which has not fully been assessed yet, most importantly the contribution of dark matter and radial gradients in the IMF. Assuming that the tension is real and not the effect of systematics, the most plausible way to reconcile model and observations is to allow the effective IMF of satellite galaxies to be drawn from a different distribution, heavier at fixed galaxy properties, than that describing centrals.

Finally, we find that the relative dependence of the IMF on stellar mass and velocity dispersion gets mixed by dry mergers, making it difficult to observationally determine the fundamental parameter(s) governing the IMF from $z \sim 0$ measurements.

\section{ACKNOWLEDGMENTS}

CN acknowledges financial support from PRIN MIUR 20102011, project The Chemical and Dynamical Evolution of the MilkyWay and Local Group Galaxies, prot. 2010LY5N2T.

\section{REFERENCES}

Auger M. W., Treu T., Gavazzi R., Bolton A. S., Koopmans L. V. E., Marshall P. J., 2010a, ApJ, 721, L163

Auger M. W., Treu T., Bolton A. S., Gavazzi R., Koopmans L. V. E., Marshall P. J., Moustakas L. A., Burles S., 2010b, ApJ, 724, 511

Barnabè M., Czoske O., Koopmans L. V. E., Treu T., Bolton A. S., 2011, MNRAS, 415, 2215

Bastian N., Covey K. R., Meyer M. R., 2010, ARA\&A, 48, 339

Bekki K., 2013, MNRAS, 436, 2254

Bezanson R., van Dokkum P., van de Sande J., Franx M., Kriek M., 2013, ApJ, 764, L8

Cappellari M., et al., 2009, ApJ, 704, L34

Cappellari M., et al., 2012, Nature, 484, 485

Cassata P., et al., 2013, ApJ, 775, 106

Chattopadhyay T., De T., Warlu B., Chattopadhyay A. K., 2015, ApJ, 808, 24

Conroy C., van Dokkum P. G., 2012, ApJ, 760, 71

Dutton A. A., Mendel J. T., Simard L., 2012, MNRAS, 422, 33

Fakhouri O., Ma C.-P., Boylan-Kolchin M., 2010, MNRAS, 406, 2267

Fontanot F., De Lucia G., Hirschmann M., Bruzual G., Charlot S., Zibetti S., 2016, preprint, (arXiv:1606.01908)

Gargiulo I. D., et al., 2015a, MNRAS, 446, 3820

Gargiulo A., Saracco P., Longhetti M., Tamburri S., Lonoce I., Ciocca F., 2015b, A\&A, 573, A110
Gavazzi R., Treu T., Rhodes J. D., Koopmans L. V. E., Bolton A. S., Burles S., Massey R. J., Moustakas L. A., 2007, ApJ, 667,176

Guszejnov D., Krumholz M. R., Hopkins P. F., 2016, MNRAS, 458,673

Hennebelle P., Chabrier G., 2011, ApJ, 743, L29

Hopkins P. F., 2012, MNRAS, 423, 2037

Hopkins P. F., Bundy K., Hernquist L., Wuyts S., Cox T. J., 2010, MNRAS, 401, 1099

Ilbert O., et al., 2013, A\&A, 556, A55

Krumholz M. R., 2011, ApJ, 743, 110

La Barbera F., Ferreras I., Vazdekis A., de la Rosa I. G., de Carvalho R. R., Trevisan M., Falcón-Barroso J., Ricciardelli E., 2013, MNRAS, 433, 3017

La Barbera F., Vazdekis A., Ferreras I., Pasquali A., Cappellari M., Martín-Navarro I., Schönebeck F., Falcón-Barroso J., 2016, MNRAS, 457, 1468

Leauthaud A., et al., 2012, ApJ, 744, 159

López-Sanjuan C., et al., 2012, A\&A, 548, A7

Martín-Navarro I., La Barbera F., Vazdekis A., Falcón-Barroso J., Ferreras I., 2015, MNRAS, 447, 1033

Mason C. A., et al., 2015, ApJ, 805, 79

McConnell N. J., Lu J. R., Mann A. W., 2016, ApJ, 821, 39

Naab T., Johansson P. H., Ostriker J. P., 2009, ApJ, 699, L178

Nagashima M., Lacey C. G., Okamoto T., Baugh C. M., Frenk C. S., Cole S., 2005, MNRAS, 363, L31

Navarro J. F., Frenk C. S., White S. D. M., 1997, ApJ, 490, 493

Newman A. B., Ellis R. S., Treu T., Bundy K., 2010, ApJ, 717, L103

Newman A. B., Treu T., Ellis R. S., Sand D. J., 2013, ApJ, 765, 25

Newman A. B., Ellis R. S., Treu T., 2015, ApJ, 814, 26

Nipoti C., Treu T., Leauthaud A., Bundy K., Newman A. B., Auger M. W., 2012, MNRAS, 422, 1714

Offner S. S. R., 2015, preprint, (arXiv:1510.06027)

Onodera M., et al., 2012, ApJ, 755, 26

Posacki S., Cappellari M., Treu T., Pellegrini S., Ciotti L., 2015, MNRAS, 446, 493

Posti L., Nipoti C., Stiavelli M., Ciotti L., 2014, MNRAS, 440, 610

Salpeter E. E., 1955, ApJ, 121, 161

Schneider M. D., Hogg D. W., Marshall P. J., Dawson W. A., Meyers J., Bard D. J., Lang D., 2015, ApJ, 807, 87

Sersic J. L., 1968, Atlas de galaxias australes. Cordoba, Argentina: Observatorio Astronomico

Shetty S., Cappellari M., 2014, ApJ, 786, L10

Smith R. J., Lucey J. R., 2013, MNRAS, 434, 1964

Smith R. J., Lucey J. R., Conroy C., 2015, MNRAS, 449, 3441

Sonnenfeld A., Nipoti C., Treu T., 2014, ApJ, 786, 89

Sonnenfeld A., Treu T., Marshall P. J., Suyu S. H., Gavazzi R., Auger M. W., Nipoti C., 2015, ApJ, 800, 94

Spiniello C., Trager S., Koopmans L. V. E., Conroy C., 2014, MNRAS, 438, 1483

Spiniello C., Barnabè M., Koopmans L. V. E., Trager S. C., 2015, MNRAS, 452, L21

Tinker J., Kravtsov A. V., Klypin A., Abazajian K., Warren M., Yepes G., Gottlöber S., Holz D. E., 2008, ApJ, 688, 709

Tortora C., Romanowsky A. J., Napolitano N. R., 2013, ApJ, 765,8

Tortora C., Napolitano N. R., Saglia R. P., Romanowsky A. J., Covone G., Capaccioli M., 2014, MNRAS, 445, 162

Treu T., Auger M. W., Koopmans L. V. E., Gavazzi R., Marshall P. J., Bolton A. S., 2010, ApJ, 709, 1195

de Vaucouleurs G., 1948, Annales d'Astrophysique, 11, 247

van Dokkum P. G., et al., 2010, ApJ, 709, 1018

van de Sande J., et al., 2013, ApJ, 771, 85

van der Wel A., Holden B. P., Zirm A. W., Franx M., Rettura A., Illingworth G. D., Ford H. C., 2008, ApJ, 688, 48 


\section{APPENDIX A: HIERARCHICAL BAYESIAN INFERENCE}

We use a similar hierarchical Bayesian inference method as the one used by S15 to fit for the distribution in IMF of the same sample of galaxies. The method can be summarized as follows. We assume that the density profile of early-type galaxies can be described by the sum of a stellar component with a de Vaucouleurs profile and a dark-matter component with an NFW (Navarro et al. 1997) profile. We parametrize this model in terms of the Salpeter stellar mass $M_{*}^{\text {Salp }}$, effective radius $R_{\text {eff }}$, projected dark-matter mass within a cylinder of radius $5 \mathrm{kpc} M_{\mathrm{DM}, 5}$, dark-matter scale radius $r_{s}$ and effective IMF $\alpha_{\mathrm{IMF}}$. Following S15 we assume that the effective radius is known exactly and we fix $r_{s}=10 R_{\text {eff }}$ for simplicity. The exact value of $r_{s}$ has little impact on lensing and dynamics measurements, more sensistive to the mass distribution at the scale of a few $\mathrm{kpc}$, much smaller than typical values of $r_{s}$ predicted by numerical simulations. The free parameters of each object are then $M_{*}^{\text {Salp }}, M_{\mathrm{DM}, 5}$ and $\alpha_{\mathrm{IMF}}$. For a more compact notation we label this triplet $\boldsymbol{\omega}$.

$$
\boldsymbol{\omega} \equiv\left(M_{*}^{\mathrm{Salp}}, M_{\mathrm{DM}, 5}, \alpha_{\mathrm{IMF}}\right) .
$$

From these parameters we calculate the model central velocity dispersion, $\sigma_{e 2}$, using the spherical Jeans equation and assuming isotropic stellar velocity distribution. Though a simplification, the spherical isotropic model has been shown to match results obtained with a more accurate dynamical modeling (Barnabè et al. 2011).

We assume that the parameters of each galaxy are drawn from a distribution for the population of galaxies, described by a set of hyper-parameters $\boldsymbol{\eta}$ :

$$
\boldsymbol{\omega}_{i} \sim \operatorname{Pr}\left(\boldsymbol{\omega}_{i} \mid \boldsymbol{\eta}\right),
$$

where the subscript $i$ indicates the $i$-th galaxy in the sample. We model this distribution as the product of four terms:

$$
\operatorname{Pr}\left(\boldsymbol{\omega}_{i} \mid \boldsymbol{\eta}\right)=\mathcal{G}_{*}\left(M_{*, i}^{\mathrm{Salp}} \mid \boldsymbol{\eta}\right) \mathcal{G}_{\sigma}\left(\sigma_{e 2, i} \mid M_{*}^{\mathrm{Salp}}, \boldsymbol{\eta}\right) \mathcal{G}_{\mathrm{DM}}\left(M_{\mathrm{DM}, 5, i} \mid M_{*}^{\mathrm{Salp}}, \sigma_{e 2, i}, \boldsymbol{\eta}\right) \mathcal{G}_{\mathrm{IMF}}\left(\alpha_{\mathrm{IMF}, i} \mid M_{*}^{\mathrm{Salp}}, \sigma_{e 2, i}, \boldsymbol{\eta}\right),
$$

where each term is a log-normal distribution respectively in $M_{*}^{\text {Salp }}, \sigma_{e 2}$ (which is a well-defined function of $\boldsymbol{\omega}$ ), $M_{\mathrm{DM}, 5}$ and $\alpha_{\mathrm{IMF}}$. More precisely,

$$
\begin{gathered}
\mathcal{G}_{*}\left(M_{*, i}^{\text {Salp }} \mid \boldsymbol{\eta}\right)=\frac{1}{\sqrt{2 \pi} \sigma_{*}} \exp \left\{-\frac{\left(\log M_{*, i}^{\text {Salp }}-\mu_{*}\right)^{2}}{2 \sigma_{*}^{2}}\right\} . \\
\mathcal{G}_{*}\left(\sigma_{e 2, i} \mid \boldsymbol{\eta}\right)=\frac{1}{\sqrt{2 \pi} \sigma_{\sigma}} \exp \left\{-\frac{\left(\log \sigma_{e 2, i}-\mu_{\sigma}\left(z_{i}, M_{*, i}^{\text {Salp }}\right)\right)^{2}}{2 \sigma_{\sigma}^{2}}\right\},
\end{gathered}
$$

where

$$
\begin{gathered}
\mu_{\sigma}\left(M_{*, i}^{\mathrm{Salp}}\right)=\mu_{\sigma, 0}+\zeta_{\sigma}\left(z_{i}-0.3\right)+\beta_{\sigma}\left(\log M_{*, i}^{\mathrm{Salp}}-11.5\right) . \\
\mathcal{G}_{*}\left(M_{\mathrm{DM}, 5, i} \mid \boldsymbol{\eta}\right)=\frac{1}{\sqrt{2 \pi} \sigma_{\mathrm{DM}}} \exp \left\{-\frac{\left(\log M_{\mathrm{DM}, 5, i}-\mu_{\mathrm{DM}}\left(z_{i}, M_{*, i}^{\mathrm{Salp}}, \sigma_{e 2, i}\right)\right)^{2}}{2 \sigma_{\mathrm{DM}}^{2}}\right\},
\end{gathered}
$$

where

$$
\begin{gathered}
\mu_{\mathrm{DM}}\left(M_{*, i}^{\mathrm{Salp}}, \sigma_{e 2, i}\right)=\mu_{\mathrm{DM}, 0}+\zeta_{\mathrm{DM}}\left(z_{i}-0.3\right)+\beta_{\mathrm{DM}}\left(\log M_{*, i}^{\mathrm{Salp}}-11.5\right)+\xi_{\mathrm{DM}}\left(\log \sigma_{e 2, i}-2.4\right) \\
\mathcal{G}_{*}\left(\alpha_{\mathrm{IMF}, i} \mid \boldsymbol{\eta}\right)=\frac{1}{\sqrt{2 \pi} \sigma_{\mathrm{IMF}}} \exp \left\{-\frac{\left(\log \alpha_{\mathrm{IMF}, i}-\mu_{\mathrm{IMF}}\left(z, M_{*, i}^{\text {Salp }}, \sigma_{e 2, i}\right)\right)^{2}}{2 \sigma_{\mathrm{IMF}}^{2}}\right\}
\end{gathered}
$$

where

$$
\mu_{\mathrm{IMF}}\left(M_{*, i}^{\text {Salp }}, \sigma_{e 2, i}\right)=a_{z}\left(z_{i}-0.3\right)+a_{*}\left(\log M_{*, i}^{\text {Salp }}-11.5\right)+a_{\sigma}\left(\log \sigma_{e 2, i}-2.4\right)+\log \alpha_{\mathrm{IMF}, 0} .
$$

Since the lenses in the S15 study are collected from two different surveys, SLACS and SL2S, we allow for different values in the mean and dispersion of the stellar mass distribution in equation $(\mathrm{A} 4): \mu_{*}^{\text {SLACS }}, \mu_{*}^{\text {SL2S }}, \sigma_{*}^{\text {SLACS }}$ and $\sigma_{*}^{\text {SL2S }}$.

In summary the distribution of the parameters describing individual galaxies, equation (A3), is described by 18 hyperparameters:

$$
\boldsymbol{\eta}=\left\{\mu_{*}^{\mathrm{SLACS}}, \mu_{*}^{\mathrm{SL} 2 \mathrm{~S}}, \sigma_{*}^{\mathrm{SLACS}}, \sigma_{*}^{\mathrm{SL} 2 \mathrm{~S}}, \mu_{\sigma, 0}, \zeta_{\sigma}, \beta_{\sigma}, \sigma_{\sigma}, \mu_{\mathrm{DM}, 0}, \zeta_{\mathrm{DM}}, \beta_{\mathrm{DM}}, \xi_{\mathrm{DM}}, \sigma_{\mathrm{DM}}, \log \alpha_{\mathrm{IMF}, 0}, a_{z}, a_{*}, a_{\sigma}, \sigma_{\mathrm{IMF}}\right\} .
$$

We wish to infer the posterior probability distribution of these hyper-parameters given the data $\mathbf{d}$. The data consists of a measurement of the Einstein radius, velocity dispersion and stellar mass from stellar population synthesis analysis for each object. From Bayes theorem,

$$
\operatorname{Pr}(\boldsymbol{\eta} \mid \mathbf{d}) \propto \operatorname{Pr}(\boldsymbol{\eta}) \operatorname{Pr}(\mathbf{d} \mid \boldsymbol{\eta})
$$


Table A1. Full model, corresponding to Fig. 2. Median and $68 \%$ limits on the posterior probability function of each hyper-parameter, marginalized over the other parameters.

\begin{tabular}{lcl}
\hline & & Parameter description \\
\hline$\mu_{*}^{\text {SLACS }}$ & $11.58 \pm 0.03$ & Mean $\log M_{*}^{\text {Salp }}$, SLACS sample \\
$\sigma_{*}^{\text {SLACS }}$ & $0.19 \pm 0.02$ & Scatter in $\log M_{*}^{\text {Salp }}$, SLACS sample \\
$\mu_{*}^{\text {SL2S }}$ & $11.50 \pm 0.05$ & Mean $\log M_{*}^{\text {Salp }}$, SL2S sample \\
$\sigma_{*}^{\text {SL2S }}$ & $0.23 \pm 0.04$ & Scatter in $\log M_{*}^{\text {Salp }}$, SL2S sample \\
$\mu_{\sigma, 0}$ & $2.39 \pm 0.01$ & Mean $\log \sigma$ at $z=0.3$ and $\log M_{*}^{\text {Salp }}=11.5$ \\
$\zeta_{\sigma}$ & $-0.01 \pm 0.03$ & Linear dependence of $\log \sigma$ on $z$ \\
$\beta_{\sigma}$ & $0.19 \pm 0.03$ & Linear dependence of $\log \sigma$ on $\log M_{*}^{\text {Salp }}$ \\
$\sigma_{\sigma}$ & $0.04 \pm 0.01$ & Scatter in $\log \sigma$ \\
$\mu_{\mathrm{DM}, 0}$ & $10.60 \pm 0.09$ & Mean $\log M_{\mathrm{DM}, 5}$ at $z=0.3, \log M_{*}^{\text {Salp }}=11.5$ and $\log \sigma=2.4$ \\
$\zeta_{\mathrm{DM}}$ & $1.13 \pm 0.27$ & Linear dependence of $\log M_{\mathrm{DM}, 5}$ on $z$ \\
$\beta_{\mathrm{DM}}$ & $0.13 \pm 0.25$ & Linear dependence of $\log M_{\mathrm{DM}, 5}$ on $\log M_{*}^{\text {Salp }}$ \\
$\xi_{\mathrm{DM}}$ & $-0.77 \pm 0.72$ & Linear dependence of $\log M_{\mathrm{DM}, 5}$ on $\log \sigma$ \\
$\sigma_{\mathrm{DM}}$ & $0.25 \pm 0.05$ & Scatter in $\log M_{\mathrm{DM}, 5}$ \\
$\log \alpha_{\mathrm{IMF}, 0}$ & $0.06 \pm 0.02$ & Mean $\log \alpha_{\mathrm{IMF}}$ at $z=0.3, \log M_{*}^{\text {Salp }}=11.5$ and $\log \sigma=2.4$ \\
$a_{z}$ & $-0.20 \pm 0.09$ & Linear dependence of $\log \alpha_{\mathrm{IMF}}$ on $z$ \\
$a_{*}$ & $0.10 \pm 0.10$ & Linear dependence of $\log \alpha_{\mathrm{IMF}}$ on $\log M_{*}^{\text {Salp }}$ \\
$a_{\sigma}$ & $0.49 \pm 0.39$ & Linear dependence of $\log \alpha_{\mathrm{IMF}}$ on $\log \sigma$ \\
$\sigma_{\mathrm{IMF}}$ & $0.03 \pm 0.01$ & Scatter in $\log \alpha_{\mathrm{IMF}}$ \\
\hline
\end{tabular}

Table A2. Model with $a_{*}=0$, corresponding to Fig. 3. Median and $68 \%$ limits on the posterior probability function of each hyperparameter, marginalized over the other parameters.

\begin{tabular}{lcl}
\hline & & Parameter description \\
\hline$\mu_{*}^{\text {SLACS }}$ & $11.59 \pm 0.03$ & Mean $\log M_{*}^{\text {Salp }}$, SLACS sample \\
$\sigma_{*}^{\text {SLACS }}$ & $0.19 \pm 0.02$ & Scatter in $\log M_{*}^{\text {Salp }}$, SLACS sample \\
$\mu_{*}^{\text {SL2S }}$ & $11.50 \pm 0.05$ & Mean $\log M_{*}^{\text {Salp }}$, SL2S sample \\
$\sigma_{*}^{\text {SL2S }}$ & $0.24 \pm 0.04$ & Scatter in $\log M_{*}^{\text {Salp }}$, SL2S sample \\
$\mu_{\sigma, 0}$ & $2.39 \pm 0.01$ & Mean $\log \sigma$ at $z=0.3$ and $\log M_{*}^{\text {Salp }}=11.5$ \\
$\zeta_{\sigma}$ & $-0.01 \pm 0.04$ & Linear $\operatorname{dependence~of~} \log \sigma$ on $z$ \\
$\beta_{\sigma}$ & $0.18 \pm 0.02$ & Linear dependence of $\log \sigma$ on $\log M_{*}^{\text {Salp }}$ \\
$\sigma_{\sigma}$ & $0.05 \pm 0.00$ & Scatter in $\log \sigma$ \\
$\mu_{\mathrm{DM}, 0}$ & $10.63 \pm 0.07$ & Mean $\log M_{\mathrm{DM}, 5}$ at $z=0.3, \log M_{*}^{\text {Salp }}=11.5$ and $\log \sigma=2.4$ \\
$\zeta_{\mathrm{DM}}$ & $1.12 \pm 0.24$ & Linear $\operatorname{dependence~of~} \log M_{\mathrm{DM}, 5}$ on $z$ \\
$\xi_{\mathrm{DM}}$ & $-0.52 \pm 0.68$ & Linear $\operatorname{dependence~of~} \log M_{\mathrm{DM}, 5}$ on $\log \sigma$ \\
$\sigma_{\mathrm{DM}}$ & $0.25 \pm 0.05$ & Scatter in $\log M_{\mathrm{DM}, 5}$ \\
$\log \alpha_{\mathrm{IMF}, 0}$ & $0.06 \pm 0.01$ & Mean $\log \alpha_{\mathrm{IMF}}$ at $z=0.3, \log M_{*}^{\text {Salp }}=11.5$ and $\log \sigma=2.4$ \\
$a_{z}$ & $-0.19 \pm 0.09$ & Linear dependence of $\log \alpha_{\mathrm{IMF}}$ on $z$ \\
$a_{\sigma}$ & $0.81 \pm 0.21$ & Linear dependence of $\log \alpha_{\mathrm{IMF}}$ on $\log \sigma$ \\
$\sigma_{\mathrm{IMF}}$ & $0.04 \pm 0.01$ & Scatter in $\log \alpha_{\mathrm{IMF}}$ \\
\hline
\end{tabular}

where $\operatorname{Pr}(\boldsymbol{\eta})$ is the prior on the hyper-parameters and $\operatorname{Pr}(\mathbf{d} \mid \boldsymbol{\eta})$ the likelihood of observing the data given the values of the hyper-parameters. This latter term is the product over all galaxies

$$
\operatorname{Pr}(\mathbf{d} \mid \boldsymbol{\eta})=\prod_{i} \operatorname{Pr}\left(\mathbf{d}_{i} \mid \boldsymbol{\eta}\right)
$$

Each term in this product can in turn be expanded as

$$
\operatorname{Pr}\left(\mathbf{d}_{i} \mid \boldsymbol{\eta}\right)=\int d \boldsymbol{\omega}_{i} \operatorname{Pr}\left(\mathbf{d}_{i} \mid \boldsymbol{\omega}_{i}\right) \operatorname{Pr}\left(\boldsymbol{\omega}_{i} \mid \boldsymbol{\eta}\right)
$$

We assume flat priors on all hyper-parameters. We then sample the posterior probability distribution with a Markov Chain Monte Carlo. The integrals in equation (A14) are evaulated via importance sampling and Monte Carlo integration following Schneider et al. (2015). The inferred values of the hyper-parameters with $1-\sigma$ uncertainties are reported in Table A1.

As described in Section 3, we then repeated the analysis by fixing $a_{*}=0$. In this second analysis we also fix $\beta_{\mathrm{DM}}=0$. The inferred values of the hyper-parameters with $1-\sigma$ uncertainties are reported in Table A2. 